\title{
Uso de DSC na Determinação de Parâmetros de Vulcanização de Látex de Borracha Natural
}

\author{
Augusto C. C. Peres, Lea M. A. Lopes, Leila L.Y. Visconte, Regina C.R. Nunes \\ Instituto de Macromoléculas Professora Eloisa Mano, UFRJ
}

\begin{abstract}
Resumo: Os processos de obtenção de artefatos de borracha natural (NR) a partir do látex e a composição dos sistemas de vulcanização são fundamentais na determinação da aplicabilidade e qualidade destes artefatos. Varias técnicas podem ser usadas para a verificação da eficiência do processamento e dos sistemas de vulcanização. O objetivo deste trabalho foi estudar a eficiência da vulcanização de látex de NR por dois sistemas de vulcanização SV1 (sistema convencional) e SV2 (sistema eficiente) em diferentes concentrações através de medidas calorimétricas. A Calorimetria Diferencial de Varredura (DSC) mostrou ser uma técnica eficaz na obtenção de parâmetros de vulcanização como velocidade, tempo de prévulcanização, bem como do excesso de ligações cruzadas. Os resultados foram corroborados pelos ensaios de resistência à tração e análise óptica. De forma geral, o sistema de vulcanização convencional SV1, em comparação ao sistema eficiente SV2 mostrou-se mais adequado para uso, nas condições experimentais deste trabalho, por fornecer maior velocidade de vulcanização, maior segurança de processo e melhor desempenho mecânico.
\end{abstract}

Palavras-chave: Vulcanização, látex de borracha natural, DSC.

\section{The Use of DSC in the Determination of Natural Rubber Latex Vulcanization}

\begin{abstract}
The manufacture of natural rubber goods from the latex has significant commercial interest. The processes to obtain these artifacts, the type and amounts of the vulcanization systems used are of utmost importance in determining their quality and suitability. A number of techniques can be used to verify the efficiency of both the processing and the vulcanization systems. The objective of this work was the study of the efficiency of NR latex vulcanization carried out by two vulcanization systems, namely SV1 (conventional system) and SV2 (efficient system) in different concentrations, by differential scanning calorimetry (DSC). The DSC has been found to be a very efficient technique for the acquisition of data which would allow the determination of the vulcanization rate, pre-vulcanization time and the excess of crosslinks in elastomers latexes compositions. The results have been corroborated by stress strength tests and optical analysis. In general, the conventional system, SV1, as compared with the efficient SV2, gave the best results, under the experimental conditions, since it provided a higher vulcanization rate, better process safety and better mechanical behavior.
\end{abstract}

Keywords: Vulcanization, Natural RubberLatex, DSC.

\section{Introdução}

O uso de borracha natural (NR) na forma de látex representa aproximadamente $13 \%$ do mercado total de NR e suas principais aplicações são em materiais cirúrgicos, preservativos, carpetes, revestimentos de materiais têxteis e $\operatorname{adesivos}^{[1]}$. Os principais processos de produção são por coagulação e por desidratação. Neste último um molde é imerso num compósito de látex concentrado e lentamente retirado sob movimentos circulares, para a redistribuição do excesso da emulsão. Em seguida este dispositivo passa pelo processo de secagem quando, pelo binômio temperatura e tempo, ocorrerá a vulcanização. Nesta etapa o artefato é retirado do molde ${ }^{[2]}$.

Os sistemas de vulcanização utilizados em látices, para que tenham aplicação tecnológica, são dispersões cuja eficiência não depende apenas dos seus componentes, mas prin- cipalmente, do tamanho de partícula e da solubilidade destes na micela do látex ${ }^{[1-4]}$. Estes sistemas consistem normalmente de três componentes: enxofre, acelerador e óxido de zinco. Muitas variações são possíveis, tais como, o uso de aceleradores doadores de enxofre em substituição ao enxofre, ou o uso de dois aceleradores para obtenção de curas mais rápidas. Idealmente todo o enxofre adicionado deve gerar ligações cruzadas, e a quantidade adicionada deve ser a mínima necessária para se alcançar as propriedades desejadas ${ }^{[2]}$.

A vulcanização de elastômeros insaturados com sistemas acelerados com enxofre é um processo químico muito complexo envolvendo reações simultâneas e consecutivas. As propriedades dos artefatos obtidos dependem da quantidade e dos tipos de ligações formadas. O enxofre elementar é convertido em uma variedade de ligações poli-sulfídicas, (mono, di e poli-sulfídicas, intraciclização e como radicais pendentes) e a distribuição destas não depende somente do tipo e da

Autor para correspondência: Augusto C. C. Peres, Instituto de Macromoléculas Professora Eloisa Mano, UFRJ, Caixa Postal 68525, CEP: 21945-970, Rio de Janeiro, RJ. E-mail: augusto@ima.ufrj.br 
quantidade do acelerador utilizado, mas também do tempo, da temperatura, da razão molar entre acelerador e enxofre e da concentração do ativador (óxido de zinco) ${ }^{[5-8]}$.

Considerando os teores de enxofre e acelerador, os sistemas de vulcanização podem ser classificados como: Eficiente (EV) - (teor de enxofre entre 0,3 a 1,0phr e de acelerador entre 2,0 a 6,0phr); Semi-Eficiente (Semi-EV) - (teor de enxofre entre 1,0 a $2,0 \mathrm{phr}$ e de acelerador entre 1,0 a 2,5phr); e Convencional (teor de enxofre entre 2,0 a $3,5 \mathrm{phr}$ e de acelerador entre 0,5 a $1,0 \mathrm{phr})$. O tipo do sistema além de influenciar as propriedades do produto final, afeta diretamente a sua processabilidade pela modificação do tempo de prévulcanização, que se traduz na prática, como o tempo máximo que a composição pode ser exposta à temperatura de processo sem que haja a formação de um teor ligações cruzadas que comprometa seu comportamento reológico e reprocessabilidade. O sistema eficiente induz a formação de ligações mono e di-sulfídicas que aumentam a resistência ao envelhecimento do artefato, reduzem o tempo de prévulcanização e são responsáveis por módulos menores. Nos sistemas convencionais onde se detecta o maior percentual das ligações poli-sulfídicas, há redução da resistência ao envelhecimento, porém promovendo maior desempenho mecânico e podendo aliar um longo tempo de pré-vulcanização com uma alta taxa de velocidade de vulcanização ${ }^{[2,6]}$. Na escolha do sistema de vulcanização, alem de se considerar as propriedades desejadas, o custo deve ser levado em conta e, neste aspecto, o sistema convencional apresenta vantagens.

A vulcanização é estudada por várias técnicas sendo uma delas a calorimetria diferencial de varredura (DSC). A comprovação da reação de cura se dá pela presença de pico exotérmico que desaparece numa segunda varredura ${ }^{[7,10]}$. No caso do DSC este pico é relacionado pela entalpia, sendo interpretada como um somatório das reações que ocorrem durante a vulcanização. Estudos sobre a vulcanização por DSC têm mostrado que, sob condições específicas de varredura, a entalpia de vulcanização pode ser relacionada linearmente com a quantidade de enxofre elementar da formulação ${ }^{[6,9-11]}$, numa faixa de $1 \mathrm{a} 3 \mathrm{phr}$. Embora a adição de acelerador desloque a entalpia de vulcanização para temperaturas menores, a entalpia total mostra-se independente da quantidade de acelerador na faixa de 0,05 a $4 \mathrm{phr}^{[10-14]}$.

Neste trabalho foi estudada a eficiência na vulcanização de látex de NR por dois sistemas de vulcanização SV1 (sistema convencional) e SV2 (sistema eficiente) através do uso do DSC. Os sistemas foram também avaliados quanto à sua resistência à tração e examinados por microscopia óptica.

\section{Experimental}

\section{Materiais e Equipamentos}

- Látex Natural (Hevea brasiliensis) de procedência da Inducompre Indústria Comércio e Prestação de Serviços, Salvador, Bahia, tendo as seguintes características: tipo 1, centrifugado, com alto teor de amônia, e $60 \%$ em sólidos totais (ASTM D 1076).
- Dispersões de uso industrial SV1 e SV2, sendo seus componentes básicos: enxofre $(\mathrm{S})$, óxido de zinco $(\mathrm{ZnO})$, dietil ditiocarbamato de zinco (ZEDC) e sistema de emulsão. O sistema de vulcanização SV1 é do tipo convencional ( $\mathrm{S}>>$ ZEDC), e o sistema SV2 é do tipo eficiente ( $<<<$ ZEDC).

- Dinamômetro Instron modelo 1101, célula de carga de $1 \mathrm{kN}$ - Instron Corporation, Boston, Massachussets, USA.

- Calorímetro Diferencial de Varredura - Perkin Elmer modelo DSC 7 - Perkin Elmer Corporation, Nornalk, Connecticut, USA.

- Microscópio óptico - Sistema Olympus modelo BX50 Olympus Optical Co. Ltd., Shibuya-Ku, Tóquio, Japão.

\section{Preparação das Composições.}

As composições foram feitas misturando ao látex de NR, 3, 6 e 12phr de cada sistema de vulcanização usado, respectivamente, SV1 ou SV2. Este procedimento foi realizado sob agitação mecânica (40rpm) durante $1 \mathrm{~h}$, na temperatura ambiente.

Um volume próximo a $50 \mathrm{~cm}^{3}$ de cada composição foi vertido em cuba nivelada $(21 \mathrm{~cm} \times 16 \mathrm{~cm} \times 3 \mathrm{~cm})$, que foi colocada em estufa com circulação forçada de ar à $40^{\circ} \mathrm{C}$. Após a secagem, as diferentes composições foram vulcanizadas na mesma estufa por 17 min à $150^{\circ} \mathrm{C}$.

O tempo de vulcanização foi determinado pela avaliação dos resultados de resistência à tração das amostras de todas as composições em diferentes tempos de exposição. O tempo de exposição de 17min foi comum a todas as composições.

\section{Comportamento Térmico na Vulcanização.}

O comportamento térmico das misturas durante o processo de vulcanização foi caracterizado em 3 amostras, no calorímetro diferencial de varredura com uma taxa de aquecimento de $10{ }^{\circ} \mathrm{C} /$ min numa faixa de temperatura de 30 a $290{ }^{\circ} \mathrm{C} \mathrm{em}$ atmosfera de nitrogênio a vazão de 40 a $50 \mathrm{~cm}^{3} / \mathrm{min}$.

\section{Propriedades Mecânicas}

A resistência à tração foi realizada em 4 amostras (cinco corpos de prova/amostra) por experimento com base na norma ASTM D412 utilizando corpo de prova tipo gravata (dumbbell) modelo $\mathrm{C}$ com espessura de $1 \mathrm{~mm}$. As condições experimentais foram: Velocidade de afastamento de $50 \mathrm{~cm} / \mathrm{min}$ e de registro de $5 \mathrm{~cm} / \mathrm{min}$.

\section{Resultados e Discussão}

A Figura 1 mostra a curva exotérmica típica obtida por DSC para o cálculo da cura, onde podem ser identificadas três temperaturas características que são: $\mathrm{T}_{0}-$ Temperatura de inicio da reação de vulcanização, Tf - Temperatura final e TM - Temperatura da entalpia máxima. Estas temperaturas são dependentes da taxa de aquecimento. Nos sistemas elastoméricos, a linha de base antes de $\mathrm{T}_{0}$ e, após Tf raramente retorna ao mesmo nível devido à capacidade calorífica do elastômero.

A Tabela 1 apresenta os resultados de DSC obtidos para as diferentes composições. A entalpia é proporcional ao teor 


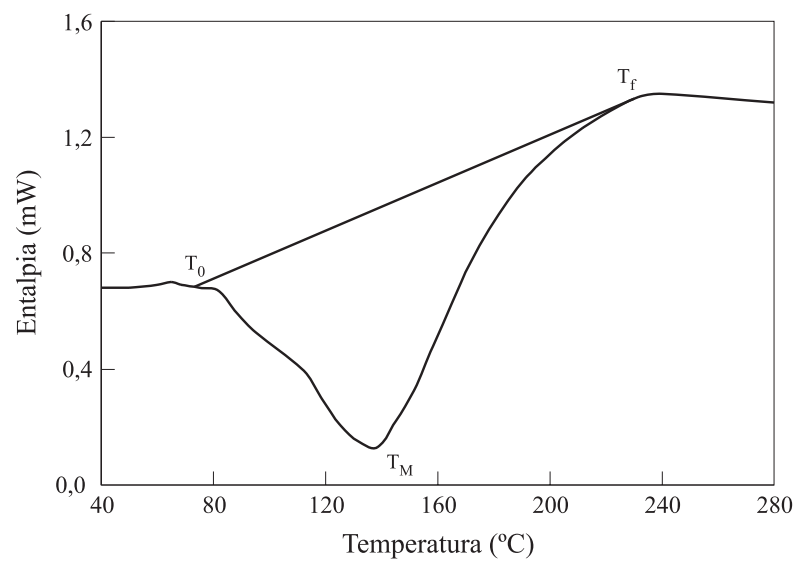

Figura 1. Curva de DSC típica para vulcanização de borracha natural com sistema de vulcanização convencional, com a velocidade de varredura de $10^{\circ} \mathrm{C} / \mathrm{min}$ com a linha de base.

Tabela 1. Parâmetros de DSC de látex de NR com os sistemas de vulcanização SV1 e SV2 nas concentrações de 3, 6 e 12 phr.

\begin{tabular}{cccccc}
\hline $\begin{array}{c}\text { Sistema de } \\
\text { Vulcanização }\end{array}$ & phr & $\begin{array}{c}\mathbf{T}_{\mathbf{0}} \\
\left({ }^{\circ} \mathbf{C}\right)\end{array}$ & $\begin{array}{c}\mathbf{T}_{\mathbf{M}} \\
\left({ }^{\circ} \mathbf{C}\right)\end{array}$ & $\begin{array}{c}\mathbf{T}_{\mathbf{f}} \\
\left({ }^{\circ} \mathbf{C}\right)\end{array}$ & $\begin{array}{c}\Delta \mathbf{H}_{\mathbf{v}} \\
(\mathbf{j} / \mathbf{g})\end{array}$ \\
\hline SV1 & 3 & 110 & 144 & 250 & 17 \\
& 6 & 90 & 136 & 245 & 42 \\
SV2 & 12 & 95 & 163 & 240 & 47 \\
& 3 & 95 & 136 & 220 & 18 \\
& 6 & 95 & 140 & 245 & 25 \\
& 12 & 95 & 141 & 245 & 45 \\
\hline
\end{tabular}

$\mathrm{T}_{0}$ e $\mathrm{T}_{\mathrm{f}}$ - Temperatura inicial e final da vulcanização, $\mathrm{T}_{\mathrm{M}}$ - Temperatura do pico exotérmico máximo e $\Delta \mathrm{H}_{\mathrm{v}}-$ Entalpia de Vulcanização.

de enxofre que forma as ligações cruzadas até o limite das ligações possíveis. Esta afirmação pode ser comprovada pelos resultados dos diferentes sistemas usados (SV1 e SV2), levando a valores de entalpia crescentes com o teor de sistema de vulcanização. Com exceção da SV1 com 6 phr de sistema de vulcanização todas as demais composições apresentam resultados compatíveis ${ }^{[6,10,12-14]}$.

O percentual de vulcanização é calculado da curva de DSC convertendo a escala de temperatura em tempo a partir de $\mathrm{T}_{0}$, que assume $o$ valor de $t=0$. As temperaturas subseqüentes até Tf são divididas pela velocidade de varredura e cada altura da curva (linha de base ate a curva) representará $\mathrm{dH} / \mathrm{dt}$.

O percentual de vulcanização é proporcional à entalpia do processo, e calculado pela seguinte equação:

$$
\% \text { Vulcanização }=\left(\Sigma \mathrm{H}(\mathrm{t}) / \Delta \mathrm{H}_{\mathrm{v}}\right) \times 100
$$

Onde: $\sum \mathrm{H}(\mathrm{t})$ - representa o somatório da entalpia em função do tempo e pode ser calculado através da soma das áreas parciais tendo como base o tempo que neste trabalho foi de 0,003 min e $\Delta \mathrm{H}_{\mathrm{v}}$ - Entalpia total de vulcanização.

A Figura 2 mostra o percentual de vulcanização em função do tempo para a taxa de aquecimento de $10^{\circ} \mathrm{C} / \mathrm{min}$ e a Tabela 2 para o tempo de 200s especificamente. O sistema SV1 apresentou um tempo de pré-vulcanização, início da mudança da inclinação da curva, em torno de 200s e SV2 em 100s.

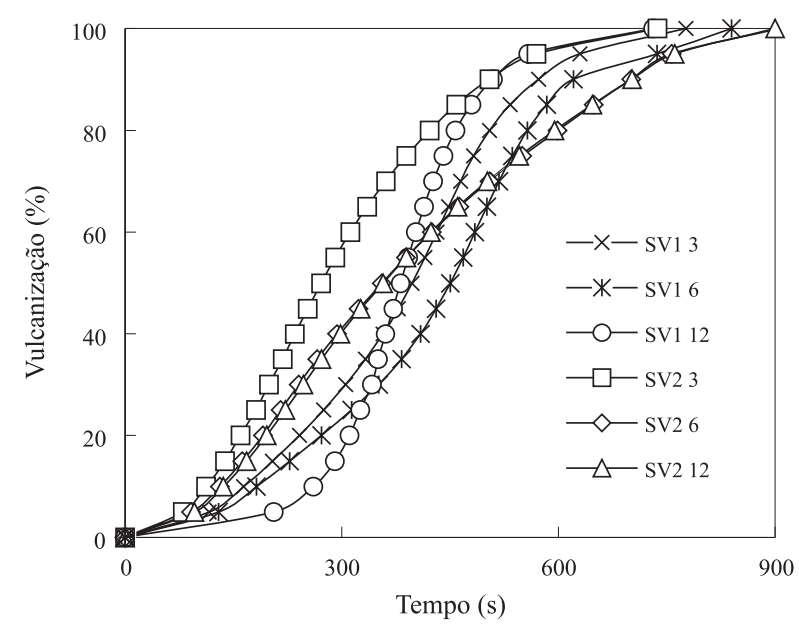

Figura 2. Percentual de vulcanização em função do tempo para uma taxa de aquecimento de $10^{\circ} \mathrm{C} / \mathrm{min}$.

Tabela 2. Taxa de velocidade e percentual de vulcanização para as composições SV1 e SV2.

\begin{tabular}{ccccc}
\hline \multirow{2}{*}{$\begin{array}{c}\text { Teor } \\
\text { (phr) }\end{array}$} & $\begin{array}{c}\text { Taxa de Velocidade (mJ/s) - } \\
\text { Conversão de } \mathbf{3 0 \%} \text { a 70\% }\end{array}$ & \multicolumn{2}{c}{$\begin{array}{c}\text { Percentual de } \\
\text { Vulcanização } \\
\text { (\%) à 200s }\end{array}$} \\
\cline { 2 - 5 } & SV1 & SV2 & SV1 & SV2 \\
\hline 3 & 0,40 & 0,38 & 15 & 30 \\
6 & 0,74 & 0,47 & 12 & 30 \\
12 & 1,78 & 0,61 & 5 & 21 \\
\hline
\end{tabular}

A velocidade de vulcanização caracterizada pela inclinação da reta entre $30 \%$ a $70 \%$ (Tabela 2), foi maior para SV1. Observa-se também que para o SV1 a velocidade aumenta em função do teor do sistema de vulcanização e em SV2 o aumento foi significativamente menor.

A Figura 3 mostra os resultados obtidos por microscopia óptica dos diferentes sistemas de vulcanização SV1 e SV2. Pela análise das micrografias é possível concluir que a dispersão dos componentes no sistema SV1 é mais homogênea, com tamanho das partículas menores do que no sistema SV2, podendo assim ser mais facilmente absorvido na micela do látex.

Esta absorção, mais eficiente, pode ser a responsável pelas propriedades finais do artefato vulcanizado.

Segundo vários autores, as propriedades mecânicas dos vulcanizados são função do número e do tipo de ligações cruzadas, havendo um limite ótimo para cada formulação ${ }^{[5-7,15]}$.

Após a vulcanização, as diferentes composições foram analisadas quanto à resistência à tração e ao alongamento na ruptura em função do teor dos sistemas de vulcanização, SV1 e SV2, mostrados nas Figuras 4 e 5. Observa-se que, para os dois sistemas de vulcanização usados, o limite para o melhor desempenho mecânico é $3 \mathrm{phr}$, com destaque para o sistema SV1. A redução da resistência à tração deve-se provavelmente ao excesso de ligações cruzadas cuja correlação já foi citada. Os valores mais baixos para o sistema SV2 em relação ao 

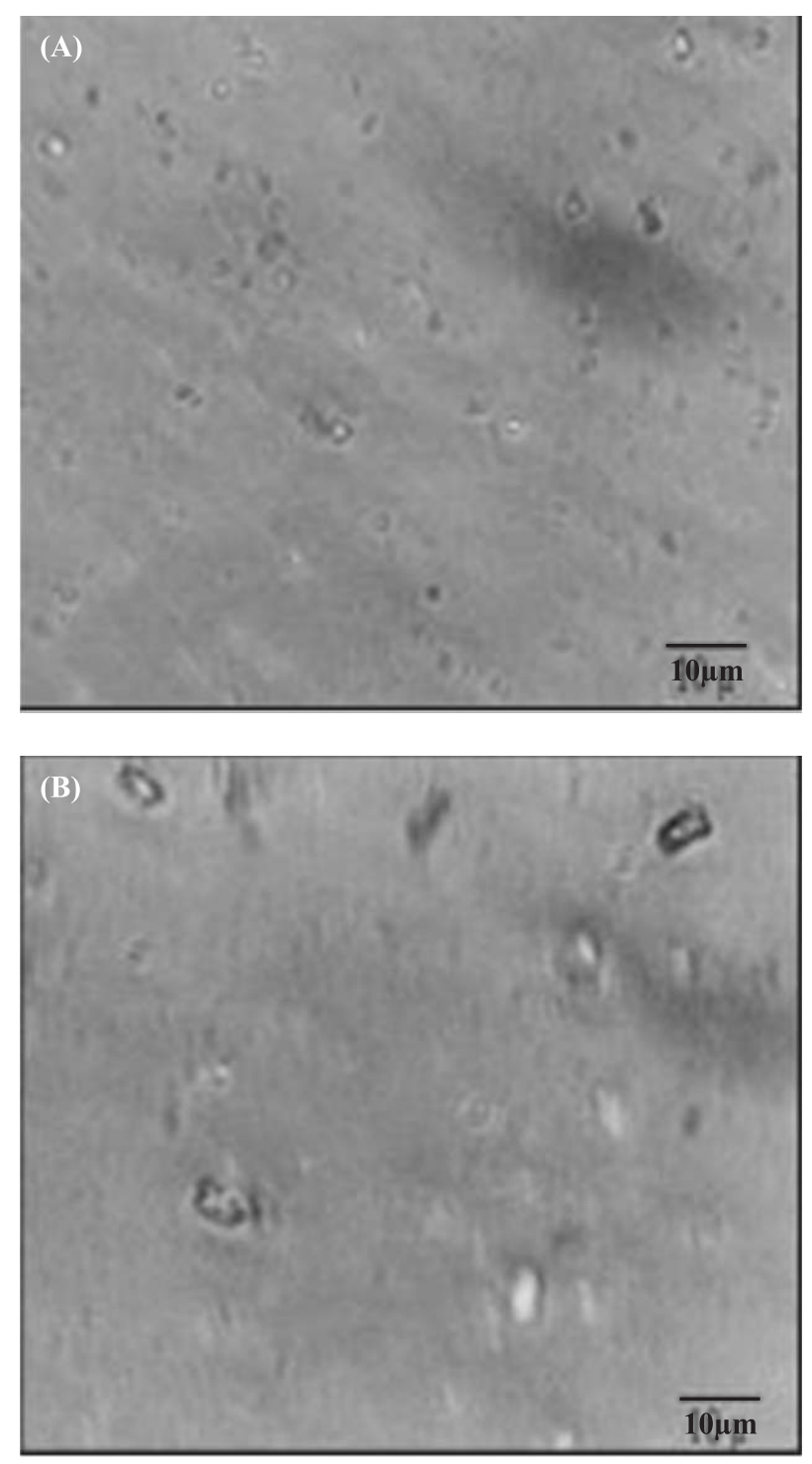

Figura 3. Micrografias dos sistemas de vulcanização, SV1 e SV2 no tempo de mistura de $1 \mathrm{~h}$ e diluída a $1 \%$. (1000X de aumento).

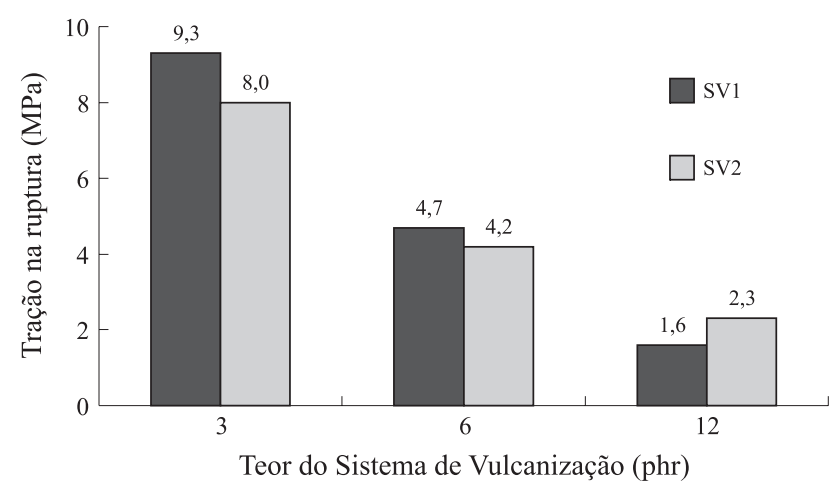

Figura 4. Resistência à tração na ruptura em função do teor dos sistemas de vulcanização.

sistema SV1 podem não estar só relacionados a sua composição (sistema eficiente), mas também com a menor homogeneidade e maior tamanho de suas partículas.

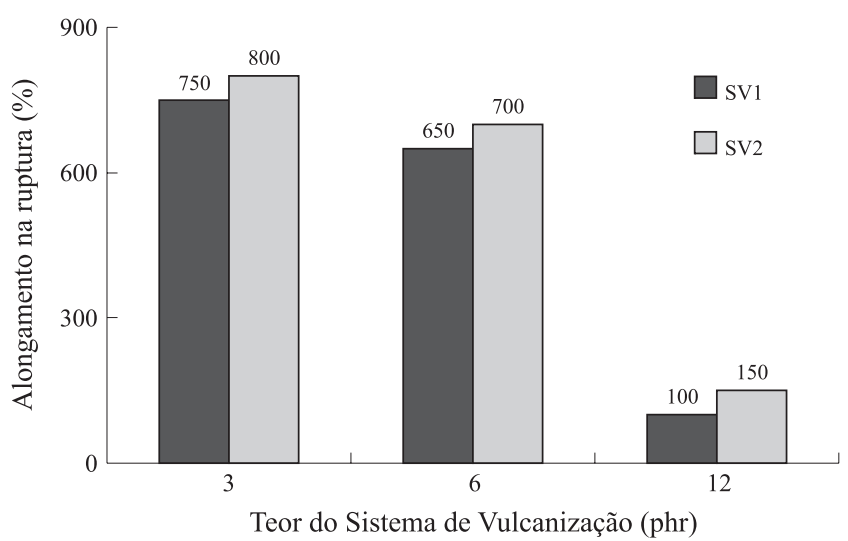

Figura 5. Alongamento na ruptura em função do teor dos sistemas de vulcanização.

Os resultados de alongamento na ruptura, mostrados na Figura 5 apresentam o mesmo comportamento observado para as medidas de resistência à tração, ou seja, um decréscimo do alongamento em função do aumento da quantidade de sistema de vulcanização. Não se observa uma diferença significativa entre os sistemas de vulcanização. A perda acentuada de alongamento nas composições com $12 \mathrm{phr}$, deve-se à restrição ao movimento das cadeias moleculares causada provavelmente pelo excesso de sistema de vulcanização.

\section{Conclusões}

A Calorimetria Diferencial de Varredura (DSC) se mostrou uma técnica eficaz para a determinação da velocidade de vulcanização, do tempo de pré-vulcanização, bem como do excesso de ligações cruzadas. Nas condições experimentais deste trabalho, o sistema de vulcanização convencional SV1 mostrou-se mais adequado, por:

- Apresentar melhor dispersão e menor tamanho de partículas

- Fornecer uma velocidade de vulcanização maior, mas com segurança de processo (maior tempo de pré-vulcanização).

- Fornecer melhor desempenho mecânico.

\section{Agradecimentos}

Os autores agradecem ao CNPq e a Fundação Universitária José Bonifácio pelo apoio financeiro.

\section{Referências Bibliográficas}

1. Peethambaran, N.R. \& Kuriakose, A.P. - Plast. Rubber Process. Applic., 11, p.179 (1999).

2. Gazeley, K.F.; Gorton, A.D.T. \& Pendle, T.D. "Technological Processing of Natural Rubber Latex", in: Natural Rubber Science and Technology, cap.4, A.D. Roberts (ed.), Oxford University Press, Suffolk (1990).

3. Rippel, M.M.; Lee, L.T.; Leite, C.A.P. \& Galembeck, F. J. Colloid and Interface Sci., 268, p.330 (2003). 
4. Garnier, B.; Danes, F. \& Delaunay, D. - Thermochim. Acta, 222, p.115 (1993).

5. Coran, A.Y. - "Vulcanization", in: Science and Technology of Rubber, cap.7, James E. Mark; Burack Erman \& Frederick R. Eirich (ed), Academic Press Inc., San Diego, 1994.

6. Chough, S.H. \& Chang, D.H. - J. Appl. Polym. Sci., 61, p.449 (1996).

7. Travas-Sejdic, J.; Jelencic, J.; Bravar, M. \& Fröbe, Z. Eur. Polym. J., 32, p.1395 (1996).

8. Rochette, B.; Sadr, A.; Abdul, M. \& Verganaud, J.M. Thermochim. Acta, 85, p.419 (1985).

9. Mathew, G. \& Kuriakose, A.P. - J. Appl. Polym. Sci., 49, p.2009 (1993).
10. Brazier, D.W. - Thermochim. Acta, 222, p.147 (1977).

11. Brazier, D.W. - Rubber Chem. Technol., 53, p.437 (1980).

12. Brazier, D.W. \& Nickel, G.H. - Rubber Chem. Technol., 48, p.26 (1975).

13. Brazier, D.W.; Nickel, G.H. \& Szentgyorgyi, Z. - Rubber Chem. Technol., 53, p.160 (1980).

14. Wang, P.Y.; Qian, H.L. \& Yu, H.P. - J. Appl. Polym. Sci., 92, p.3260 (2004).

15. Nasir, M. \& Teh, G.K. - Eur. Polym. J., 24, p.733 (1988).

Enviado: 03/08/05 Reenviado: 14/11/05 Aprovado: 23/11/05 